\title{
Parental stress during COVID-19: A brief report on the role of distance education and family resources in an Italian sample
}

\author{
Ughetta Moscardino $^{1}$ (D) $\cdot$ Raffaele Dicataldo $^{1} \cdot$ Maja Roch $^{1} \cdot$ Maria Carbone $^{1} \cdot$ Irene C. Mammarella $^{1}$
}

Accepted: 2 February 2021 / Published online: 13 February 2021

(C) The Author(s), under exclusive licence to Springer Science+Business Media, LLC part of Springer Nature 2021

\begin{abstract}
Since the COVID-19 outbreak, school closures have affected over 1.5 billion children worldwide. Many countries implemented a rapid transition to distance education (DE), but the effects of such transition on family life remain largely underexplored. The current study used a cross-sectional, correlational survey design to explore the role of DE and family resources (parenting selfefficacy and family functioning) in perceived stress among Italian parents of first-grade children $(N=89)$. Results of hierarchical multiple regression indicated that, after controlling for stressful events experienced during school closure, parents' difficulty to manage children's DE was positively linked to levels of stress. However, this association became nonsignificant after adding family resources to the model, with more parental self-efficacy and good family functioning predicting less perceived stress. The findings underscore the importance of supporting positive resources within the family environment to reduce DE-related parental stress in the context of the ongoing COVID-19 pandemic.
\end{abstract}

Keywords Distance education $\cdot$ Parental stress $\cdot$ Family resources $\cdot$ Children $\cdot$ COVID-19

\section{Introduction}

Extensive school closures during COVID-19 have affected over 1.5 billion children worldwide (UNESCO, 2020). Consequently, many countries engaged in a rapid transition to distance education (DE). Emerging evidence suggests that families struggled to manage their offsprings' remote school activities due to a lack of technological resources, their own workload, exposure to stressful events, and full-time caregiving responsibilities (Cluver et al., 2020; Golberstein, Wen, \& Miller, 2020). In particular, some studies suggest that homeschooling during the first wave of the pandemic was related to more negative parent-child interactions and lower levels of affective well-being among parents and children in different countries (Thorell et al., 2021). These difficulties were partly due to the need for parents to take on a novel teaching role involving the management of multiple tasks (e.g., structuring of children's learning activities, technological support, supervision of home assignments) that often exceeded the available resources, resulting in high levels of

Ughetta Moscardino

ughetta.moscardino@unipd.it

1 Department of Developmental Psychology and Socialization, University of Padova, Via Venezia 8, 35131 Padova, Italy stress (Schmidt, Kramer, Brose, Schmiedek, \& Neubauer, 2020). Yet, little is known about the role of resilience-related aspects in shaping parents' responses to school lockdowns caused by the COVID-19 outbreak.

Here, we used a family resilience perspective (Prime, Wade, \& Browne, 2020; Walsh, 2016) to highlight the contribution of positive aspects to parents' response to adversity. Specifically, we focused on parental self-efficacy and family functioning (Gavidia-Payne, Denny, Davis, Francis, \& Jackson, 2015). Parental self-efficacy refers to a parent's belief in his/her ability to perform the parenting role successfully (Jones \& Prinz, 2005) and represents a major source of resilience under stressful circumstances. For instance, prior research has documented associations of parental self-efficacy with greater family well-being due to the preserved ability to provide competent, quality parenting even when faced with challenges and adversity (Crnic \& Ross, 2017; Torres Fernandez, Schwartz, Chun, \& Dickson, 2013). Family functioning is a dynamic concept encompassing affective involvement, affective responsiveness, behavioral control, communication, problem solving, and roles (Epstein, Baldwin, \& Bishop, 1983). Although these aspects can be disrupted in situations of distress, they may also serve as resilience factors. Indeed, positive interactions among family members, supportive relationships, and cohesion have been found to be conducive to less parenting stress and better family quality of life in 
the context of disasters (Günther-Bel, Vilaregut, Carratala, Torras-Garat, \& Pérez-Testor, 2020; McDermott \& Cobham, 2012).

In sum, although extant research highlights substantial negative psychosocial effects of the COVID-19 pandemic on children and families (Fontanesi et al., 2020; Spinelli, Lionetti, Pastore, \& Fasolo, 2020), the contribution of family resilience to parental stress during school closure is still unclear. The current study aimed to explore the role of parenting selfefficacy and healthy family functioning in levels of perceived stress over and above the potentially detrimental effects of parental difficulties with their children's DE, after controlling for the amount of stressful events experienced during school lockdown.

\section{Method}

\section{Design and Participants}

We used a cross-sectional, correlational survey design. The sample consisted of 89 parents (79 mothers, 10 fathers) with at least one child attending first grade $\left(M_{\text {age }}=6.4\right.$ years; $S D=.30)$ recruited via contacts with primary schools in Padua, a middle-sized town in northeastern Italy.

\section{Materials and Procedure}

Data were collected online between April and June 2020, when Italian schools were closed due to a strict nationwide lockdown. Participants completed a set of questionnaires to measure each of our study variables.

Stressful Life Events We used an adapted version of the List of Threatening Experiences Questionnaire (LTE-Q; Brugha \& Cragg, 1990) to assess parents' stressful experiences in the past six months (e.g., 'death of loved ones', 'major physical injury'), integrated by four additional COVID-19 specific items (e.g., 'fear of contracting COVID-19'). Parents were asked to report whether they had experienced each of these events using a dichotomous scale $(1=$ yes, $0=$ no), with a higher score indicating exposure to more stressful experiences. The LTE-Q has good test-retest reliability, high agreement between participant and informant ratings, and good agreement with interview-based ratings (Brugha \& Cragg, 1990), whereas internal reliability is modest (Motrico et al., 2013). In the current sample, Cronbach's Alpha was .58.

$D E$ - Parents' overall difficulty with managing their children's activities related to DE was measured via an ad-hoc developed single item ('Have you ever encountered any difficulties in managing your child's DE?'), with response options ranging from 0 (never) to 5 (very often).
Parenting Self-Efficacy The 7-item Achievement subscale of the Self-Efficacy for Parenting Tasks Index (SEPTI; Coleman \& Karraker, 2000) was used to measure the extent to which parents felt they were able to follow their child's academic activities (e.g., 'I do an adequate job helping my child with school work'). This questionnaire is designed for parents of children aged 5 to 12 years. Items are rated on a six-point scale ( $1=$ strongly disagree, $6=$ strongly agree), with higher scores indicating greater perceived self-efficacy. The SEPTI has shown satisfactory internal consistency and concurrent validity with other measures of parenting self-efficacy (Coleman \& Karraker, 2000; Whittaker \& Cowley, 2006). In this sample, Cronbach's Alpha was .70.

Family Functioning We used the 12-item General Functioning subscale of the Mc Master Family Assessment Device (FAD; Epstein et al., 1983) to assess overall perceptions of family functioning. The questionnaire measures structural, organizational, and transactional characteristics of families. It comprises 6 items reflecting healthy family functioning (e.g., 'In times of crisis we can turn to each other for support') and 6 items reflecting unhealthy family functioning (e.g., 'There are lots of bad feelings in our family'). Items are rated on a 4-point scale (from $1=$ strongly agree to $4=$ strongly disagree), with higher scores indicating worse levels of family functioning after reversing the negatively worded items (Epstein et al., 1983). For ease of interpretation, we reversed the positive items so that higher scores reflected healthier family functioning. Abundant research has reported good psychometric properties of the FAD in nonclinical, psychiatric, and medical samples as well as in different countries (Staccini, Tomba, Grandi, \& Keitner, 2015), including Italy (Roncone et al., 1998). In our study, Cronbach's Alpha was .92.

Parental Stress The 10-item Perceived Stress Scale (PSS; Cohen, Kamarck, \& Mermelstein, 1983) measured the extent to which parents perceived aspects of their life as uncontrollable, unpredictable, and overloading. Participants were asked how often they felt a certain way within the past month (e.g., 'How often have you felt that you were on top of things?') using a 5-point scale from 0 (never) to 4 (very often), with higher scores indicating greater perceived stress. The PSS has demonstrated good psychometric properties in terms of internal reliability, test-retest reliability, and concurrent validity (Lee, 2012), also in Italian samples (Mondo, Sechi, \& Cabras, 2019). In the current study, Cronbach's Alpha was .72 .

Data were collected online with Qualtrics. Participants provided their informed consent. The survey took approx. $20 \mathrm{~min}$ to complete. The study was approved by the Ethic Committee of the University of Padova (protocol n. 3555). 


\section{Results}

Descriptive statistics and correlations among study variables are reported in Table 1.

A three-stage hierarchical multiple regression was conducted with perceived parental stress as the dependent variable. The sum of stressful life events was entered at stage one to control for the amount of negative events experienced by families during the past few months. Difficulty with children's DE was entered at stage 2 , and the two family resource variables (i.e., parenting self-efficacy and family functioning) at stage 3 .

The results (see Table 2) showed that, after controlling for stressful life events, difficulties with managing DE was a significant predictor of perceived parental stress (Step 2). However, when parental self-efficacy and family functioning were included in the model (Step 3), this association was no longer significant. In the final model, increased self-efficacy and healthy family functioning significantly contributed to lower levels of parental stress.

To evaluate if the assumptions of our regression model were met, we used a procedure recommended by Peña \& Slate (2006) via the gvlma package of the statistical software $\mathrm{R}$ (R Core Team 2020). In particular, we evaluated assumptions of linearity, homoscedasticity, uncorrelatedness, and normality on the residuals of our final regression model. The Global test indicated that the assumptions of the regression model could not be rejected, $\chi^{2}(4)=.410 ; p=.98$ (skewness: $\chi^{2}(1)=.001, p=.970$; curtosis: $\chi 2(1)=.051, p=.821$; link function: $\chi^{2}(1)=.093, p=.760$; homoscedasticity: $\left.\chi^{2}(1)=.264, p=.607\right)$. Thus, our model met the assumptions required to perform regression analysis.

\section{Discussion}

Given the ongoing public health emergency worldwide, understanding the ways in which parents' perceived stress is influenced by the management of children's DE and family resources is essential to plan effective intervention strategies capitalizing on extant assets and protective factors (Prime et al., 2020).
Table 2 Summary of hierarchical regression analysis for variables predicting parental stress $(N=89)$

\begin{tabular}{lllllll}
\hline Variable & $\beta$ & $t$ & $\mathrm{SE}$ & $\mathrm{R}$ & $\mathrm{R}^{2}$ & $\Delta \mathrm{R}^{2}$ \\
\hline Step 1 & & & & .34 & .11 & .11 \\
$\quad$ Sum of stressful life events & .34 & $3.19^{* *}$ & .28 & & & \\
$\quad$ Step 2 & & & & .42 & .18 & .07 \\
$\quad$ Sum of stressful life events & .28 & $2.71^{* *}$ & .28 & & & \\
$\quad$ Difficulty with children's DE & .25 & $2.43^{*}$ & .52 & & & \\
Step 3 & & & & .58 & .33 & .15 \\
Sum of Stressful life events & .27 & $2.75^{* *}$ & .26 & & & \\
$\quad$ Difficulty with children's DE & .04 & .43 & .54 & & & \\
Parenting self-efficacy & -.25 & $-2.34^{*}$ & .11 & & & \\
Family functioning & .27 & $-2.39^{*}$ & .10 & & & \\
\hline
\end{tabular}

$* p<.05, * * p<.01 . D E$ distance education

Our findings revealed that, after controlling for the number of stressful events experienced during school closure, parents' difficulty with managing their children's remote schooling was positively associated with perceived stress, supporting previous reports which highlight the potentially adverse effects of parents taking over the role of teachers on family well-being during school lockdown (Schmidt et al., 2020; Thorell et al. 2021). However, our analyses also showed that when family resources were added to the model, this association became nonsignificant. Thus, parental self-efficacy and family functioning explained levels of perceived stress over and above parents' overall difficulties with managing young children's DE, lending support to the view that these variables may be considered as key indices of family resilience that can be promoted in pandemic-related emergencies (GavidiaPayne et al., 2015). A possible explanation is that parents' self-efficacy is related to an enhanced sense of competence which, in combination with a healthy family functioning characterized by open communication, shared goals/values, reciprocal support, and problem-solving skills, positively contributes to coping adaptively with problems or setbacks, ultimately resulting in less perceived stress (Crnic \& Ross, 2017; Prime et al., 2020). Overall, this pattern confirms the importance of focusing on specific family resources that can be the targets of intervention programs aiming to boost the wellbeing of the entire family system in times of forced homeschooling.
Table 1 Descriptive statistics and correlations among study variables $(N=89)$

\begin{tabular}{|c|c|c|c|c|c|c|}
\hline & 2 & 3 & 4 & 5 & $\mathrm{M}(\mathrm{SD})$ & Range \\
\hline 1. Sum of stressful life events & $.24 * *$ & .02 & -.22 & $.30 * *$ & $5.7(1.8)$ & $3-12$ \\
\hline 2. Difficulty with children's DE & & $-.35^{* *}$ & $-.42 * *$ & $.28 * *$ & $2.8(1.1)$ & $0-5$ \\
\hline 3. Parenting self-efficacy & & & $.43 * *$ & $-.40 * *$ & $23.9(4.6)$ & $13-35$ \\
\hline 4. Family functioning & & & & $-.46^{* *}$ & $20.5(5.2)$ & $13-36$ \\
\hline 5. Perceived parental stress & & & & & $18.1(4.9)$ & $6-30$ \\
\hline
\end{tabular}

$* p<.05 ; * * p<.01 . D E$ distance education 
Alongside this novel contribution to the literature on parental stress and resilience during COVID-19, we acknowledge several caveats that need to be considered when interpreting the results, such as the limited sample size, the cross-sectional design, and the exclusive focus on first-grade children. Moreover, the use of a single-item measure to assess parents' difficulty with managing DE and the lack of information on children's academic problems prior to school closure limit the conclusions that can be drawn. However, our study provides initial evidence for parents' capability to deliver competent parenting to their children who were forced to interrupt their school path despite the overall difficult situation. Thus, during public health emergencies, strengthening support and positive competences may aid parents to view themselves as active agents, contributing to overall family quality of life.

Data Availability The dataset analyzed during the current study is available from the corresponding author on reasonable request.

\section{Declarations}

Conflict of Interest On behalf of all authors, the corresponding author states that there is no conflict of interest.

\section{References}

Brugha, T. S., \& Cragg, D. (1990). The list of threatening experiences: The reliability and validity of a brief life events questionnaire. Acta Psychiatrica Scandinavica, 82(1), 77-81.

Cluver, L., Lachman, J. M., Sherr, L., Wessels, I., Krug, E., Rakotomalala, S., Blight, S., Hillis, S., Bachman, G., Green, O., Butchart, A., Tomlinson, M., Ward, C. L., Doubt, J., \& McDonald, K. (2020). Parenting in a time of COVID-19. Lancet, 395, e 64

Cohen, S., Kamarck, T., \& Mermelstein, R. (1983). A global measure of perceived stress. Journal of Health and Social Behavior, 24, 386 396.

Coleman, P. K., \& Karraker, K. H. (2000). Parenting self-efficacy among mothers of school-age children: Conceptualization, measurement and correlates. Family Relations, 49, 13-24.

Crnic, K., \& Ross, E. (2017). Parenting stress and parental efficacy. In K. Deater-Deckard \& R. Panneton (Eds.), Parental stress and early child development (pp. 263-284). Cham: Springer.

Epstein, N. B., Baldwin, L. M., \& Bishop, D. S. (1983). The McMaster family assessment device. Journal of Marital and Family Therapy, 9(2), 171-180.

Fontanesi, L., Marchetti, D., Mazza, C., Di Giandomenico, S., Roma, P., \& Verrocchio, M. C. (2020). The effect of the COVID-19 lockdown on parents: A call to adopt urgent measures. Psychological Trauma Theory Research Practice and Policy, 12(S1), S79-S81.

Gavidia-Payne, S., Denny, B., Davis, K., Francis, A., \& Jackson, M. (2015). Parental resilience: A neglected construct in resilience research. Clinical Psychologist, 19(3), 111-121.

Golberstein, E., Wen, H., \& Miller, B. F. (2020). Coronavirus disease 2019 (COVID-19) and mental health for children and adolescents. JAMA Pediatrics, 174(9), 819-820.
Günther-Bel, C., Vilaregut, A., Carratala, E., Torras-Garat, S., \& PérezTestor, C. (2020). A mixed-method study of individual, couple, and parental functioning during the state-regulated COVID-19 lockdown in Spain. Family Process, 59(3), 1060-1079.

Jones, T. L., \& Prinz, R. J. (2005). Potential roles of parental self-efficacy in parent and child adjustment: A review. Clinical Psychology Review, 25(3), 341-363.

Lee, E. H. (2012). Review of the psychometric evidence of the perceived stress scale. Asian Nursing Research, 6(4), 121-127.

McDermott, B. M., \& Cobham, V. E. (2012). Family functioning in the aftermath of a natural disaster. BMC Psychiatry, 12(1), 1-7.

Mondo, M., Sechi, C., \& Cabras, C. (2019). Psychometric evaluation of three versions of the Italian perceived stress scale. Current Psychology, 1-9.

Motrico, E., Moreno-Küstner, B., de Dios Luna, J., Torres-González, F., King, M., Nazareth, I., et al. (2013). Psychometric properties of the list of threatening experiences-LTE and its association with psychosocial factors and mental disorders according to different scoring methods. Journal of Affective Disorders, 150(3), 931-940.

Peña, E. A., \& Slate, E. H. (2006). Global validation of linear model assumptions. Journal of the American Statistical Association, 101(473), 341-354.

Prime, H., Wade, M., \& Browne, D. T. (2020). Risk and resilience in family well-being during the COVID-19 pandemic. American Psychologist, 75(5), 631-643.

Roncone, R., Rossi, L., Muiere, E., Impallomeni, M., Matteucci, M., Giacomelli, R., Tonietti, G., \& Casacchia, M. (1998). The Italian version of the family assessment device. Social Psychiatry and Psychiatric Epidemiology, 33(9), 451-461.

$\mathrm{R}$ Core Team (2020). $R$ : A language and environment for statistical computing. R Foundation for Statistical Computing, Vienna, Austria. https://www.Rproject.org.

Schmidt, A., Kramer, A. C., Brose, A., Schmiedek, F., \& Neubauer, A. B. (2020). Homeschooling and affective well-being of parents and children during the COVID-19 pandemic: A daily diary study. PsyArXiv. https://doi.org/10.31234/osf.io/sntxz.

Spinelli, M., Lionetti, F., Pastore, M., \& Fasolo, M. (2020). Parents' stress and Children's psychological problems in families facing the COVID-19 outbreak in Italy. Frontiers in Psychology, 11, 1713.

Staccini, L., Tomba, E., Grandi, S., \& Keitner, G. I. (2015). The evaluation of family functioning by the Family Assessment Device: A systematic review of studies in adult clinical populations. Family Process, 54(1), 94-115.

Thorell, L. B., Skoglund, C. B., de la Peña, A. G., Baeyens, D., Fuermaier, A., Groom, M., et al. (2021). Parental experiences of homeschooling during the COVID-19 pandemic: Differences between seven European countries and between children with and without mental health conditions. European Child and Adolescent Psychiatry. https://doi.org/10.1007/s00787-020-01706-1.

Torres Fernandez, I., Schwartz, J. P., Chun, H., \& Dickson, G. (2013). Family resilience and parenting. In D. S. Becvar (Ed.), Handbook of family resilience (pp. 119-136). New York: Springer.

UNESCO (2020). COVID-19 impact on education. Retrieved from https://en.unesco.org/covid19/educationresponse

Walsh, F. (2016). Family resilience: A developmental systems framework. European Journal of Developmental Psychology, 13(3), 313-324.

Whittaker, K. A., \& Cowley, S. (2006). Evaluating health visitor parenting support: Validating outcome measures for parental self-efficacy. Journal of Child Health Care, 10(4), 296-308.

Publisher's Note Springer Nature remains neutral with regard to jurisdictional claims in published maps and institutional affiliations. 